\title{
Audience Role in Mathematical Proof Development
}

\author{
Zoe Ashton
}

Received: date / Accepted: date

\begin{abstract}
The role of audiences in mathematical proof has largely been neglected, in part due to misconceptions like those in Perelman \& OlbrechtsTyteca (1969) which bar mathematical proofs from bearing reflections of audience consideration. In this paper, I argue that mathematical proof is typically argumentation and that a mathematician develops a proof with his universal audience in mind. In so doing, he creates a proof which reflects the standards of reasonableness embodied in his universal audience. Given this framework, we can better understand the introduction of proof methods based on the mathematician's likely universal audience. I examine a case study from Alexander and Briggs's work on knot invariants to show that we can fruitfully reconstruct mathematical methods in terms of audiences.
\end{abstract}

Keywords Argumentation · Mathematical Practice - Proof · Deduction · Formal Derivation · Audience · Knot Theory

\section{Introduction}

In A Treatise of Human Nature, David Hume noted that "there is no Algebraist nor Mathematician so expert in his science, as to place entire confidence in any truth immediately upon his discovery of it ... Every time he runs over his proofs, his confidence encreases; but still more by the approbation of his friends; and is rais'd to its utmost perfection by the universal assent and applauses of the learned world" Hume(1739), p. 231]. Hume's account points to a social context in which proofs are developed. The mathematician's confidence in his proof increases as new audiences assent to the conclusion and his

Zoe Ashton

Dept. of Philosophy, Ohio State University

230 N Oval Mall, Columbus, OH 43210, USA

Tel.: 614-292-3663

E-mail: ashton.95@osu.edu 
confidence reaches its highest point when he receives universal assent. When the learned world assents to his conclusion, he is most confident in its truth. Although recent work has focused more on the social contexts in which mathematics is practiced ${ }^{1}$ there is almost no research on the role of audiences in mathematical proof development ${ }^{2}$

In this paper, I focus on the role audiences play in the mathematician's attempt to ensure universal assent. In Section 2, I will show that, contrary to Perelman and Olbrechts-Tyteca(1969), mathematical proof is an argumentation that reflects audience consideration. Section 3 focuses on identifying which audience is involved in proof development. To close the discussion, I present a case study from knot theory in Section 4. I argue that we can fruitfully reconstruct parts of mathematical proof in terms of the mathematician's considered audiences.

\section{Mathematical Proof as Audience-Reflective Argumentation}

While informal logic has been successfully adopted in the study of mathematical proof, almost none of this research involves audiences ${ }^{3}$ This lack of focus on audiences stems from a claimed distinction between mathematical proof and argumentation, where argumentation is a three part system consisting of a premise set, conclusion, and chain of reasoning which reflect the audience considered during construction ${ }^{4}$ In this section, I argue that mathematical proof is an argumentation. 5 In Section 3. I will examine which audience is envisioned and how it is involved in the process of proving. For now, I focus on establishing that mathematical proofs reflect consideration of an audience.

\footnotetext{
1 See for example $\operatorname{Larvor(2016a)}$.

2 The most closely relevant example would be Dufour(2013) which, although it deals with similar argumentation literature, only looks at audience role in mathematical argumentation outside of proof.

3 For a collection on mathematics and argumentation theory, see Aberdein and Dove(2013)]. In that collection alone, very few papers discuss audiences and, when they do, it is related to presentational changes after the completion of a proof. After proof completion is one of the core parts of Dufour(2013)]. None of the papers examine the role of audiences in development of a proof.

4 There are various uses and definitions of "argumentation." Some authors, like Corcoran(1989)], define argumentation as a three part system comprised of a premise set, conclusion, and chain of reasoning. Others, like Tindale(2015), include the social processes of giving and taking reasons in their definition; they usually reserve argument for the name of the product devoid of process. In choosing my definition, I attempt to adhere more closely to Corcoran(1989) and view the argumentation as the product. To his definition, I add that an argumentation is a product which reflects the consideration of the audience. This makes it possible to encompass the argumentation that Perelman and Olbrechts-Tyteca(1969) are concerned with while examining their distinction between "argumentation" and "demonstration." Under definitions without an audience-reflective component, both "demonstrations" and "argumentations" would be types of argumentation.

5 The "proof" discussed in this paper is not the object of what logicians call proof theory. Rather, I intend to encompass those proofs that mathematicians comfortably write and read. It is the proof typical of mathematical practice. They are found in textbooks, journals, and seminars. I intend it to be interpreted as a broad class.
} 
The goals of my argument here are not unique. Many have argued in a similar vein to arrive at the core claim that mathematical proofs are not usually derivations. This is probably one of the more dominant views in philosophy of mathematics today ${ }_{6}^{6}$ And yet, while this claim is well-argued for, and accepted, it has not yet been shown that these arguments are compatible with a specific type of rhetorical theory, one which focuses on the role of audience. This theory, based in Perelman and Olbrechts-Tyteca's (1969) The New Rhetoric explicitly bars logical and mathematical proof. But an appropriate investigation of the audience role in proof, just as in typical argumentation, will need to deal with works influenced by The New Rhetoric. The general claim in 2.2 is broad and accepted. But the argument for the particular claim, one that bridges a heavy amount of technical jargon in both fields, is new and necessary for my arguments in the following sections.

\subsection{Argumentation and Derivation}

I begin this section with a discussion of argumentations in general. Define an argumentation as a three-part system comprised of premises, a conclusion, and a chain of reasoning, where the chain of reasoning reflects audience consideration. Each inference in the chain of reasoning is a component argument, where argument is a two-part system comprised of premises and a conclusion. The goal of giving an argumentation is to convince an audience that the truth of the conclusion follows from the premises 7 For an argumentation to successfully convince an audience, the chain of reasoning must be comprised of component arguments that the audience accepts, a premise set that the audience accepts, and the conclusion must match the intended one. If the component arguments are not acceptable to an audience, then the argumentation will not convince that audience of the truth of the conclusion. Some arguments are immediately validated - they require no chain of reasoning for an audience to be convinced that the conclusion is true, given the premises.

Perelman and Olbrechts-Tyteca(1969) contrast argumentation with what they call "demonstration." They argue that for mathematical proof, audience consideration is not reflected in the chain of reasoning because it is not an argumentation. For them, modern formal logic is, "the study of the methods of

\footnotetext{
6 For similar arguments, see Dove(2009), Aberdein(2019), and Larvor(2016b). All these have arguments to the effect that mathematical proof is not usually derivation. But the question usually involves accepting this fact and then asking whether or not mathematical proof is reducible to derivation which is clear in examples like Azzouni(2004)], Azzouni(2009), Tanswell(2015). It is also assumed in discussions like Antonutti Marfori(2010)], Larvor(2016b)], Carrascal(2015)] which are all interested in determining the best approach to rigor in proof, given that they're not typically derivations.

7 The "follows from" here is broadly construed. In many normal argumentations, we would be happy with the truth following with a certain probability. In addition, conclusions could follow from emotional appeal. In mathematical proof the truth of the theorem should follow with certainty. This is not to say that they only do so by deduction, as many proof methods

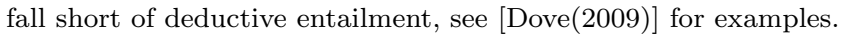


demonstration used in the mathematical sciences" Perelman and OlbrechtsTyteca(1969), p. 10]. The "demonstration" consists only of those component arguments that are instances of the rules of inference or axiom schemas. Typically, logic textbooks refer to "demonstrations" as deductions, derivations, or formal derivations; I adopt 'derivation' for the rest of the paper. What Perelman and Olbrechts-Tyteca say about logic is, according to their definition, carried through to mathematical proof as well 8 The defining characteristic of derivation is that it can be mechanically checked to determine if it is valid. The component arguments of a derivation can be immediately validated in the system. If it turns out that an argument is not immediately validated, one can argue about including that argument in the system. But this would be argumentation outside of the derivation. A derivation includes only those arguments determined to be immediately validated beforehand. Because of this, audience consideration does not play a main role in the choice of component argument in the derivation - any derivation should convince any audience of the truth of the conclusion and the validity of the argument, given the set of rules.

In contrast to derivation, Perelman and Olbrechts-Tyteca(1969) claim that all other argumentation necessarily reflects the audience whose assent the arguer sought. There is not necessarily an agreed upon set of immediately validated arguments for argumentation. If an argumentation is constructed in accordance with the intended audience's standards for component arguments and premises, then the argumentation is far more likely to succeed. So component arguments are chosen with this in mind. Moreover, an argumentation cannot be constructed without at least some audience influence on the choice of component argument. Whether this audience influence is successfully applied - i.e. did the arguer use component arguments that the audience actually accepts or just ones he thought they would accept - is a further problem. While a derivation must convince it uses only immediately validated, agreed upon inference rules. Argumentations allow more freedom in component arguments since they can have component arguments which are not among an explicit set of inference rules. These component arguments need not be deductive at all - inference to the best explanation, arguments by analogy, etc. Given this, there is an incredible range of choices when constructing an argumentation. And this range is best navigated by making one's choices in accordance with one's audience. There is still choice within derivation - what to assume in a reductio, which order to apply rules in, etc. But since these are mechanically checkable, previously agreed upon rules their choice is not guided by the reasons one thinks an audience will accept. Rather, these component arguments in derivations reflect the rule set, not the audience. Any choice within the set of rules will be such that the audience accepts it. This is because accepting

8 The barring of audiences from derivation is continued in modern rhetorical argumentation theory and follows in Perelman and Olbrechts-Tyteca(1969)'s tradition. See, for example, Tindale(2015) p. 63] which claims that a "[derivation] aims to derive conclusions from strict premises. In these terms it is an altogether different enterprise to argumentation, which involves, essentially, a meeting of minds." 
a component argument involves judging that component argument as reasonable.

In the derivation case, the component arguments were already accepted as part of the system. In the argumentation case, the available component arguments could involve any inference, which could convince the audience that the conclusion is true. When one's choices expand to rules outside a mechanically checkable set, one should choose in accordance with one's target audience. An arguer aims to choose which of those component arguments are acceptable to that audience. At base, the choice of component argument in an argumentation depends on what the arguer believes about the intended audience. And because the choice depended on the arguer's audience consideration, the final product, an argumentation, reflects this audience consideration.

\subsection{Mathematical Proofs are not Typically Derivations}

I've identified two conditionals from Perelman and Olbrechts-Tyteca(1969)]. If a mathematical proof is a derivation, then it doesn't reflect the arguer's consideration of intended audience. If a mathematical proof is an argumentation, then it must necessarily reflect the arguer's consideration of intended audience. The standing arguments that proofs are not usually derivations is not enough to warrant the claim that proofs are the argumentations that Perelman and Olbrechts-Tyteca(1969) is concerned with. Once that is shown, we can claim that proof necessarily reflects the intended audience. As the previous arguments show, audiences are often, but not necessarily, considered and affect how mathematicians write. In what follows, I argue that typical mathematical proof is not deduction, by considering a potential test for identifying mathematical derivations and showing that typical mathematical proof fails that test. Recall that derivations can be mechanically checked and the allowable component arguments are established before a derivation is constructed ${ }^{9}$ These decisions are made explicitly and, if they change, they change for all derivations in that system. Proof checking systems like Mizar have the same characteristics. They have sets of axioms and sets of immediately validated component arguments. If a typical mathematical proof is a derivation, then it should be, upon translation into the proper language, verifiable by proof checking systems.

I will test this using an example of a mathematical proof in Mizar given by Wiedijk(2004)]. He gives a number of mathematical proofs and their Mizarverified counterparts with an intermediate step called a formal proof sketch. A formal proof sketch is a Mizar article with only justification errors. A Mizar article is a text file containing a proof in the Mizar language. This article can be submitted to Mizar to check for errors. One type of error that Mizar checks for is a justification error on a line which indicates that there is not enough justification in Mizar's allowable inferences for that step. Formal proof

9 See Perelman and Olbrechts-Tyteca(1969), p. 13] for more about "demonstrations." 


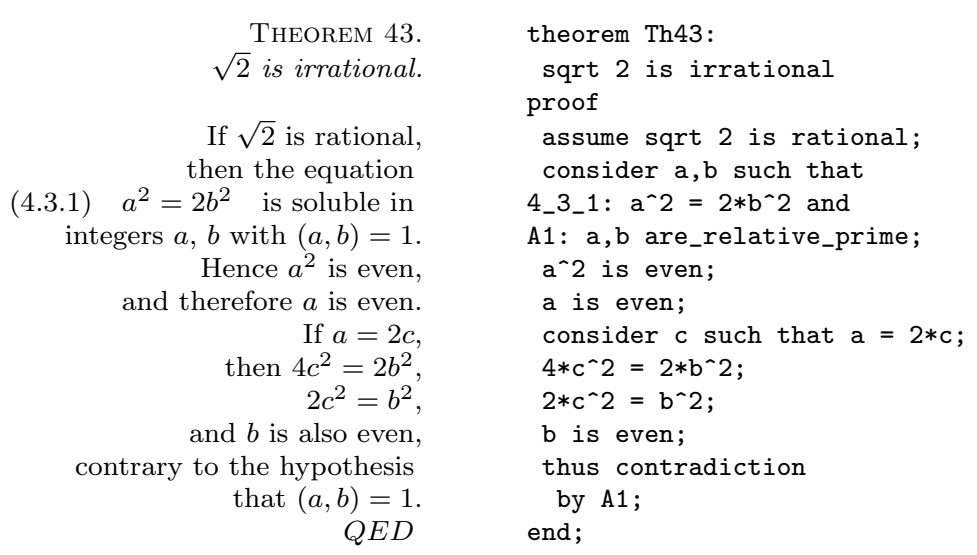

Fig. 1 The Mizar formal proof sketch is on the right. The textbook proof is on the left.

sketches can be made into full Mizar articles by "adding steps, and references between the steps, to the proofs" Wiedijk(2004), p. 379]. Wiedijk's example - a textbook proof of the $\sqrt{2}$ is irrational - is reproduced in Figure 1 next to its formal proof sketch in Mizar. Looking at Figure 1, it's clear that the Mizar formal proof sketch is a straight translation of the mathematical proof into Mizar's language. The textbook proof is a mathematical proof and no more justification is needed for the mathematician to assent to the conclusion. There is even a note concerning the naming of formal proof sketches since "these formal sketches are designed to follow the informal proofs of mathematics, and that mathematicians consider those to be proofs, and not sketches" Wiedijk(2004), p. 383]. But the Mizar formal proof sketch returns eight justification errors for the last eight lines. These justification errors indicate that there is a justificatory difference between the derivation and the mathematical proof. Mathematicians assent to the conclusion with the gaps between component arguments ${ }^{10}$ Mizar will not accept those same component arguments. For comparison with what is acceptable, Wiedijk's complete Mizar proof is reproduced in 2 .

The textbook version of the proof is a typical case of mathematical proof. The Mizar example shows that typical mathematical proofs, as they stand, are not derivations. To become derivations, they must be altered by using different component arguments ${ }^{11}$ The justification gaps show that, even by Perelman

\footnotetext{
10 For more on acceptable gaps in mathematical proof, see Fallis(2003).

11 Some argue that proofs are derivations with the "obvious" steps left out. This is compatible with the argument above. I make no claim about whether or not all mathematical proofs could be made derivations. There may be a system which makes all mathematical proofs into derivations without changing the mathematical proof itself. But it suffices for my argument that mathematicians do not need that system. They convince each other with component arguments in their mathematical proof that succeed because they reflect a consideration for that audience. This is true even of positions like Azzouni(2004)'s where a proof is taken to indicate a derivation. These proofs are still reflective of their audiences
} 


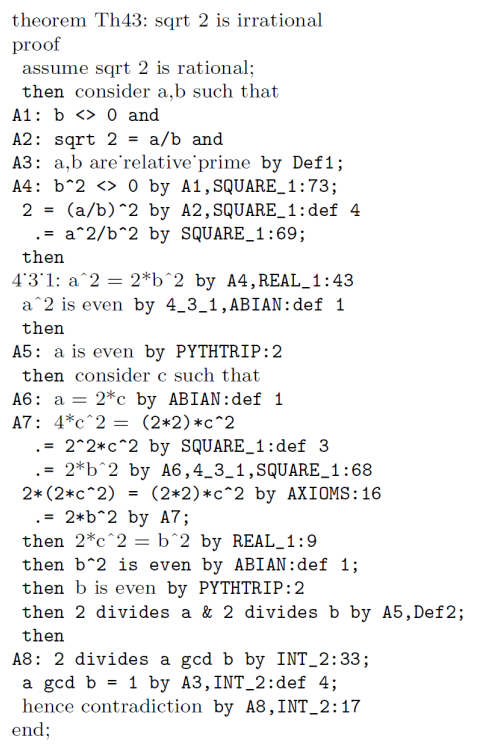

Fig. 2 The Mizar complete version of Pythagoras' proof.

and Olbrechts-Tyteca's definition, mathematical proofs are not derivations. While the goal of giving a proof already indicated this, the gaps between component arguments, the lines with justification errors, confirms this under the Perelman and Olbrechts-Tyteca definition. For them, gaps are only allowed in argumentations, not derivations. And since all argumentations necessarily reflect audience consideration, in the goals and the component arguments, proof must do so. The component arguments that the mathematician chooses are dependent on what he believes the audience will take as immediately validated.

Some distinct features of mathematical proof involve the types of immediately validated arguments. Mathematicians, unlike typical arguers, do attempt to avoid certain ethotic or pathotic component arguments. Mathematical proofs are also different than everyday argumentations in the fact that they endorse at least some formalization. Everyday arguers have no desire or interest in making formal any part of their arguments. But mathematicians often find local formalizations useful and interesting. The distinctive nature of proof should be by no means lost by this discussion. But the fact that typical proof is argumentation does lead us to the fact that it must necessarily be influenced by an intended audience. The question remains - what audience is it that is being considered during proof development? In the next section I will argue that the audience usually involved in development is the universal audience.

since they are argumentations. It is a problem outside the scope of this paper as to whether or not the derivations that they indicate is also audience-reflective. 


\section{Construction of Argumentation for the Universal Audience}

In the last section, I argued that typical mathematical proofs are argumentations. Therefore, they must reflect their intended audience. This section identifies the likely candidate for the intended audience. The concept most usually associated with the term "audience" is a particular audience. In this section, I will argue that the mathematician primarily argues to another type of audience called the universal audience while proving ${ }^{12}$ The modifier "universal" is used since it is defined by what the arguer thinks are universal standards of reasonableness. The goal of arguing to the universal audience is to create an argumentation all reasonable persons will accept. This, I will argue, is the intended audience in proof development.

More specifically, the universal audience allows us to reconcile (a) the mathematician's belief in universality of his proof with (b) the inherently audience relative nature of argumentation. To some extent the following argument proceeds by eliminating plausible particular audiences in favor of the universal audience. In addition, it draws further support from the fact that the majority of feedback received during the development of proof comes from incarnations of the universal audience. This is in line with the overarching theory of how the universal audience is employed. In the next section, I'll explore an example of the concrete ramifications of this framework in mathematical proof.

\subsection{Goals of Proof and Description of Process}

One of the most effective ways to determine the intended audience of an argumentation is to look at the goals the arguer has. So to begin this section, we should look to mathematician's description of their goals and processes. These will set the boundaries by which we decide whether or not a proposed audience is compatible with mathematical practice. One important consideration which reveals information about what audience mathematical proof development is concerned with, involves the goals of proof. Much like argumentation in other areas, the goal of giving an argumentation can help identify who it is the arguer wants to convince. Many mathematicians claim that what they do when investigating a problem is attempting to understand the mathematical concepts or relations between them. The argumentation, or proof, serves this goal in that it deepens or clarifies their understanding by showing that certain facts - those proved theorems - are true.

Moreover, one of the goals of proof is that it cements the truth of theorems forever. This can be seen in the way mathematicians talk about the reasons for proving something. For example, Stephen Krantz writes that:

\footnotetext{
12 The universal audience was first introduced in Perelman and Olbrechts-Tyteca(1969). The version I discuss here mainly follows Tindale(2015)]. For Perelman and OlbrechtsTyteca(1969)], it is never clear if the universal audience actually physically exists or if one could access it. Tindale(2015) argues that the universal audience must be a representation defined by the standards of reasonableness abstracted from particular audiences.
} 
Mathematical assertions that are proved according to the accepted canons of mathematical deduction are believed to be irrefutably true. And they will continue to be true. This permanent nature of mathematics makes it unique among human intellectual pursuits. Krantz(2011). p. 8]

Here, we can see that proof is meant to ensure permanence. G. H. Hardy also provides us with information about what the goal of proof is. His famous description is that:

I have always myself thought of a mathematician as in the first instance an observer, a man who gazes at a distant range of mountains and notes down his observations. His object is simply to distinguish clearly and notify to others as many different peaks as he can. There are some peaks which he can distinguish easily while others are less clear. He sees A sharply, while of B he can obtain only transitory glimpses. At last he makes out a ridge which leads from $\mathrm{A}$, and following it to its end he discovers that it culminates in B. B is now fixed in his vision, and from this point he can proceed to further discoveries. In other cases perhaps he can distinguish a ridge which vanishes in the distance, and conjectures that it leads to a peak in the clouds or below the horizon. But when he sees a peak he believes that it is there simply because he sees it. If he wishes someone else to see it, he points to it, either directly or through the chain of summits which led him to recognise it himself. When his pupil also sees it, the research, the argument, the proof is finished. $\operatorname{Hardy}(1929)$, p. 18]

Hardy himself recognizes this to be only a sketch of how he imagines proof works. But the goal is clear - to point directly or through the chains of summits until the pupil sees the peak. It seems unlikely that 'pupil' here is restricted only to cases of explaining well-known results to students. Hardy seems to be describing something common to research proof as well, which can be evidenced by his later claim that "the image gives us a genuine approximation to the processes of mathematical pedagogy on the one hand and of mathematical discovery on the other" $\operatorname{Hardy}(1929)$, p. 18]. So the method of proof, for Hardy, is pointing which aims at getting some other to see the peak. At the risk of stretching the analogy too far, pointing is not particular to mathematics. Mathematical proof involves maneuvers analogous to very simple procedures for guiding attention.

Moreover, while the terminology and concepts are distinctly mathematical, the reasoning process which is meant to guide a proof ought to be universal as well. Hadamard, in reflecting on how to teach mathematical reasoning and proof development heuristically, comes to the following conclusion:

In reality, the mistake is to believe that there is a trick, a slight of hand of some sort ... The nature of our questions, in my opinion, is something else and very simple: they should be those that the student 
asks himself, those which, learning from this experience, he asks himself a second time.

What these questions should be, how one should understand how to ask them, - not only the teacher but the student as well, that is what I have tried to outline elsewhere.

I tried, in other words to discern the rules, that all mathematicians unconsciously follow when they reason, at least the main ones; those rules that we find essentially the same in most of the examples. They are on the other hand common sense rules - truisms so to speak. Hadamard(2004), p. 40]

Hadamard seems to think that these rules governing mathematical reasoning, the sort that would feed into the accepted canons of mathematical deduction and the things that help us point, are universal truisms. They're closer to common-sense than they are to magic. The reasoning doesn't seem, upon reflection, to differ much from the way common-sense thinking works. Of course the concepts being reasoned about are very different than those involved in our common discourse. But the reasoning steps themselves are similar to the common-sense rules.

There are two points about my interpretation worth making more explicit. The first is the use of 'unconsciously.' The reasoning involved in developing a proof often includes both unconsciously respected standards and consciously respected standards. Hadamard seems to be interested in the first kind. Audience consideration might seem to involve only the second kind. While audience consideration is easiest to conceive of as a conscious process, it's not clear that it couldn't also be done unconsciously. Hadamard's view on mathematical invention heavily involves unconscious processes which are discussed in [Hadamard(1945)]. For Hadamard, the unconscious involves much mathematical work including the combination of ideas and evaluations of beauty and usefulness so as to suggest the best of these to the conscious Hadamard(1945). p. 30]. These are delicate considerations that the unconscious must undertake. Moreover, they are no less delicate than audience consideration in choosing which ideas to present to the conscious. Given this, the type of rules unconsciously followed could still involve audience considerations, much like beauty considerations. Likewise, if the unconsciously followed rules hold a requirement of generality, then what is presented to the conscious will have been influenced by this requirement and so further conscious reasoning will be affected by this requirement. So even though Hadamard is discussing unconsciously respected rules, these unconsciously respected rules can (a) involve audience consideration and (b) affect what the conscious processes can eventually precise. Given this, Hadamard's claims can still provide evidence toward the claim that the reasoning involved in mathematics is, upon reflection, general or universal truisms.

The second point involves a different interpretation of Hadamard's quote ${ }^{13}$ The alternate interpretation is that Hadamard is making the exact opposite

13 I'm grateful to an anonymous referee for pointing out this alternative interpretation. 
point - what is common in mathematical reasoning is just the universal truisms and the commonalities are therefore not productive to investigate. Mathematicians all reason in different ways and all that's common to them are the most basic truisms. But given the discussion preceeding the claim about truisms, it seems unmotivated. Hadamard thinks we can use his reflections on reasoning in order to choose appropriate questions for teaching students mathematics. What we should reveal to students via questioning is the way that they should be reasoning. And since this is the case, it would seem that Hadamard endorses the common-sense aspect as being a useful way of reasoning about mathematics, not as being the only common part of mathematical reasoning.

This discussion about the interpretation of Hadamard's claims also leads to another issue. Namely that just because Hadamard, Hardy, etc said X about mathematics, all mathematicians must believe $\mathrm{X}$. This is almost certainly not true. But these reflections provide more extreme versions of common views. Hadamard thought that the main examples of mathematical reasoning were truisms. Does that mean that any mathematician looking at her own proof thinks each step is a truism? Probably not. But it seems plausible that there's some identifiable core of Hadamard's claim which she would agree with. Namely that when she consciously thinks about the rules constraining her reasoning, these rules seem to be fairly general and obvious; they constrain her reasoning elsewhere and are the way one should reason. The same is true of the other quotes - they articulate an extreme view which is instantiated in the less discipline-reflective mathematician.

Given this, I want to pick out a common thread which is somewhat less extreme than any particular view held here. This common thread is something more mathematicians might agree with and can help identify the type of audience involved in development ${ }^{14}$ Namely, proof seems to be general, in two main ways: (1) once given, it should provide permanent truths and (2) the methodology involves what seem to the mathematician to be commonly useful methods, like common-sense rules or, analogously, pointing. This second generality is particularly important for the following discussions. The methods of reasoning and the rules involved don't seem to mathematicians to be unique to mathematics. Of course, understanding the reasons involved or recognizing the summits as summits is going to require extensive mathematical knowledge. But the directing itself is not a uniquely mathematical phenomenon. In what follows, I argue that these claims help identify the audience involved in proof development as the universal audience.

\subsection{Particular Audiences}

The mathematician's reflections just reviewed are not enough to fully tell us which audience they consider while proving. They simply relate goals, analo-

\footnotetext{
14 How many mathematicians agree with this is an empirical question. Given the interpretive issues and the evidence that quotes provide, an empirical study into this identified thread is worthwhile. The arguments here can provide the basis for such an investigation.
} 
gies, and reflections on reasoning. But these provide constraints on what audience should be attributed to proof development. In this subsection, I will argue that, while it seems prima facie plausible that particular audiences could fill this role, these constraints eliminate them as candidates.

Perelman and Olbrechts-Tyteca(1969)] are not entirely clear about how to differentiate kinds of audiences. According to [Sigler(2015)], which synthesizes the views and comments Perelman makes in later work as well, there are primarily three types of audiences which arise in Perelman's work. The first are the real audiences. These are the physical audiences which receive and react to argumentations. Next are particular audiences which are a construct based on the real audiences that the arguer wishes to persuade. Finally, there are universal audiences which are also constructs. Particular and universal audiences are constructs which guide the arguer in developing the argument. I will leave discussion of universal audiences to the next subsection.

A particular audience is some audience which is constructed, at least for argumentative purposes, in terms of some characteristics each individual shares. Examples of particular audiences include a classroom of students, a book club, a senate, etc. Typically particular audiences correspond to real audiences that the arguer wants to persuade. But one can imagine a particular audience given a set of characteristics. Imagine persuading an audience composed solely of noseless painters to buy a particular brand of paint. Perhaps you've never encountered such an audience. But, given the parameters, you'd probably not employ component arguments involving the smell of the paint. When the arguer attempts to persuade these groups, he takes into account the characteristic features of these groups and their knowledge bases ${ }^{15}$ The arguer constructs the particular audience in his mind and decides what component arguments to give based on how that constructed audience reacts to certain reasons.

Even when the arguer has access to his real audience, he still constructs a particular audience for it. He does so because the particular audience gives him a method of obtaining feedback during construction instead of presentation. His choices of component arguments are supposed to be ones he thinks the real audience will accept. One way of checking if these arguments are acceptable is to give them to the audience and see how they react. But this is inefficient since successful argumentations would rarely be constructed without constant audience participation. His particular audience can constantly participate and it is used as if it were actually an audience responding. These constructions based on real audiences give the arguer a heuristic for determining how his argumentation will be received by the actual audience.

With this in mind, we can quickly eliminate one type of audience as a candidate for mathematician's audience in proof development. The real audience is not an option for use in developing a proof. This is simply because a real, external audience doesn't constantly participate in proof development.

\footnotetext{
15 In Perelman and Olbrechts-Tyteca(1969), persuading is associated with particular audiences while convincing is associated with the universal audience. I follow their usage here. But nothing vital to my argument hangs on the technical differences between persuading and convincing.
} 
That being said, real audiences will be useful for testing a proof. And they can do so in two ways. The first would be to check if a proof is correct. The second is to check if it is convincing. Constructed audiences cannot check if a proof is correct. Their focus is on whether or not a component argument is acceptable. When it's acceptable, the component argument convinces that the conclusion is true. Checking for correctness is determining whether or not the conclusion is actually true. While these hopefully go together, and certainly do in a truth-preserving deduction, they can come apart in argumentation. An audience may find a component argument acceptable, i.e. it convinced them that the conclusion is true by an inference they find reasonable, without actually making the conclusion true. When one checks for correctness, one uses different tests and ways of thinking about whether or not certain claims are true or whether a component argument is fallacious. This is something difficult to do with a constructed audience, since they are built to identify acceptable or convincing arguments.

This helps to highlight an important difference between mathematical proof and other types of argumentation. In everyday argumentation, checking for correctness is not so prominent. Convincing, on grounds which may not entail the truth of the conclusion, is perfectly acceptable in everyday argumentation. Sometimes, convincing is all there is to everyday argumentation in politics, law, etc. But the nature of mathematical proof calls for more. Take for example, Hardy's claim that "our object is both to exhibit the pattern and to obtain assent. We cannot exhibit the pattern completely, since it is far too elaborate; and we cannot be content with mere assent from a hearer" $\operatorname{Hardy}(1929), \mathrm{p} .19]$. So proof aims to not just convince, but to convince of things that are true by use of reliable patterns. This requires that the inferential schemes called for in mathematical proof produce correct results and are applied correctly. The focus of this paper lies in accounting for how the goal of securing assent affects proof development at a very base level. But this is not intended to eliminate the importance of exhibiting logical patterns. Ensuring these logical patterns are exhibited and applied is the focus of checking for correctness.

Having eliminated real audiences as the audience involved in proof development, we can turn to some particular audiences ${ }^{16}$ The first would be a particular audience constructed of people like the mathematician. But if this is the case, the mathematician is attempting to convince other mathematicians like himself. These people would be ones trained in similar environments with similar concepts of rigor. Mathematicians now are different than those in the past and different from those of the future. This comes in tension with the goals of proof being permanent. Convincing people like oneself does not introduce any notion of permanence - mathematicians like oneself are not around

\footnotetext{
16 The potential particular audiences I explore here by no means enumerate all potential particular audiences. But they are representative of a number of suggestions and objections raised against the view. These few examples should suffice to highlight the underlying problem in trying to model proof development on particular audiences.
} 
forever ${ }^{17}$ The second particular audience would be journal reviewers. Again, journal reviewers have vastly different standards and desires across journals. This fact does not square well with the claim that the basic reasoning involved in proof development is general.

There is no doubt that consideration of journal reviewers has an effect on proof. But this effect is primarily in presentation, where presentation is meant to be understood as a stage in which one prepares a proof for other real people to evaluate it. The stages of development and presentation cycle back and forth - working up a proof for presentation often leads to more development. While development and presentation are intertwined, there is a very intuitive notion of "working the proof up for X." This is, roughly, how I define the presentation stage here and it must occur after having a proof to "work up." Having developed some proof and felt that the understanding is exhibited, the mathematician then turns to even more practical considerations related to publication and dissemination which involve particular audience consideration. These can involve a number of changes to the proof. But these happen at the presentation stage and not the development stage. Moreover, when these changes happen, the final, fully-developed, published proof would reflect a number of considered audiences including the reviewers. But this does not mean that journal reviewers are the primary audience of the development stage. They are primary in the "working up" stage. ${ }^{18}$ The journal reviewer, then, is not the primary audience in development, as I have defined development here ${ }^{19}$ Moreover, the journal reviewer, like the audience of oneself, is not around forever. They fail universality - Euclid certainly didn't care about journal reviewers.

There's one final audience suggestion that is worth examining before turning to the universal audience. This is the audience of oneself. According to this suggestion, mathematicians are trying to convince themselves alone in proof development. This is one of the strongest particular options, in part because so much of the real audiences a mathematician encounters while developing a proof is himself. But the problem of permanence and generality arise. If the mathematician's goals involve convincing others, then he is considering an audience larger than himself in development. Recall Hardy's claim that the proof wasn't done until the pupil saw the peak as well. This sort of description is

\footnotetext{
17 The one outright exception I've encountered is in Davis and Hersh(1986). They explicitly state that the goal is convincing people like the mathematician. So accepting this particular audience is not impossible or contradictory but it falls short of the reasons discussed above.

18 Some evidence for this can be found in Line Andersen(Forthcoming) which describes the process of turning a PhD student's proof into a publishable research paper. The PhD student who is experienced in mathematics but inexperienced in publishing, produces a proof which has little concern for what the journal reviewers will be convinced by. The experienced mathematician helps to improve the proof's presentation so as to increase chances of publication.

19 I stress again, that this is not a full answer to the issue regarding journal reviewers. This is because I have differentiated development and presentation in a preliminary manner. A full account, which I have no intention of giving here, should be able to make sense of the interplay between development and presentation. I hope to explore this in future work.
} 
not compatible with a story of development that focuses only on convincing oneself.

Each one of these particular audiences was a seemingly promising option given mathematical practice. But each one of them runs up against issues of generality and permanence. Particular audiences don't, in general, satisfy the goals and descriptions of proof development that were discussed in 3.2 To be clear, the claim is not that only audiences which result in genuinely permanent or general proofs will fit the role in development. The claim is that only audiences which the mathematician conceives of as accepting only general and permanent rules will fit the role. In 3.3 I will offer a constructed audience which does overcome these issues.

\subsection{The Universal Audience}

This subsection focuses on the universal audience. I will argue that this is the audience involved in proof development. To start, I give an overview of the universal audience so as to dispel concerns about its nature. I primarily follow Tindale(2015)]'s refinement of [Perelman and Olbrechts-Tyteca(1969)]'s universal audience. I then give two arguments supporting the claim that mathematical proof development is aimed at the universal audience. The first is that it matches the goals and processes described in 3.1 . The second is that the real audiences most prevalent in proof development are incarnations of the universal audience 20

Sometimes arguers want to construct argumentations that they think will convince every reasonable person ${ }^{21}$ An arguer can never check with every reasonable person to see if some component argument will be acceptable. Constructing particular audiences was an important part of building any argumentation. But it is all the more important for the universal audience, which has no accessible real counterpart. This is when arguers must argue to the universal audience. It is assumed by the arguer that the universal audience be a representation of all persons. It may not actually represent all people since the arguer abstracts standards of reasonableness from his experiences with

\footnotetext{
20 'Incarnations' is the term employed by Perelman and Olbrechts-Tyteca(1969). An incarnation of the universal audience should be understood as the real audiences whose primary reasons for being convinced are in line with the universal audience's.

21 Reasonable person and reasonableness are loaded terms. I would like to remain as neutral as possible on discussions of what is and is not reasonable. Perelman and OlbrechtsTyteca(1969)] are coming from a background in law and so the terms carry, to some extent, a legal notion. Perelman and Olbrechts-Tyteca(1969) and Tindale(2015) both seem to take reasonableness as a learned feature. There are no theoretical definitions of reasonable by which arguers judge audiences or people. Given a certain set of experiences, one may come to bar unreasonable people, see for example discussions in 12.3 of Tindale(2015). A full account of reasonableness is outside the scope of this paper. For present purposes it should suffice that mathematicians do make judgments of reasonableness. Moreover, this discussion deserves extensive discussion in the future, as it seems to me that the reflective arguer should be allowed to place certain meta-theoretic conditions on reasonableness to help further target his audience.
} 
particular audiences. Because each arguer encounters different sets of particular and real audiences, each arguer will have a different universal audience. What is the relationship between real, particular, and universal audiences? Since the universal audience is a constructed audience, it can be constructed out of both real audiences and particular audiences. The particular audiences one constructs can influence what one thinks a reasonable person might accept. Real people also influence what one thinks a reasonable person might accept.

It may be argued that the universal audience is a misnomer in two ways. The first is that the universal audience is not universal. This is true in a number of ways. It does not include every person. Nor is the constructed universal audience the same for each person. But its universality comes out in its goals. The goal of arguing to the universal audience is to create an argumentation that any person would accept, were they capable of understanding the reasons provided. Here 'any' is as broad as possible. It includes people from other disciplines, other times, other traditions, etc. The idea is that the component arguments would be acceptable to all these people, if they were in the appropriate language with definitions that they understood. From the view of argumentation theory there are many universal audiences - one for each arguer. But for the arguer there is only one universal audience. This is why it is called a universal audience.

At this point, many readers will doubt that Andrew Wiles' proof of Fermat's Last Theorem is written toward any reasonable person. The concerns for this are at first quite compelling. The language and concepts in Wiles' proof are simply not for everyone. Of all the reasonable people, very few of them have the training or understanding needed for reading Wiles' proof. So, as the objection goes, it seems contradictory that Wiles' argumentation is one that aims at the assent of any reasonable person. But it will be helpful to recall Hadamard(2004)]'s claim that the reasons being employed in mathematics were common-sense or truisms. Hadamard is drawing on the fact that, while the concepts and the language of mathematical proof are different, the reasoning involved seems close to common-sense. Common-sense reasoning, by definition, is something that all reasonable persons share, or at least ought to share.

The other way universal audience could be charged as a misnomer is in calling it an audience. Universal audiences are characterized by standards of reasonableness abstracted from the particular audiences an arguer encounters. Why not then say that universal audiences are a set of standards? I would like to offer a response that involves an analogy to how representations of particular audiences are constructed and what role they play in argumentation development. Arguers abstract key characteristics from particular audiences in order to effectively persuade them. Say an arguer is trying to persuade a group of mothers. His experiences with mothers in the past gives him enough information to construct a representation of the particular audience. We continue to call this representation an audience because of how it is used. The arguer doesn't simply have a set of rules about component arguments given 
to mothers. He uses the representation to test potential argumentations in his head before settling on one he thinks will persuade the particular audience. The method for abstracting and employing the representation of a universal audience is no different than for particular audiences. It is done by recognizing the underlying reasonableness in particular and real audiences. The difference in universality is that the arguer assumes all people are reasonable according to standards he's seen. He does not so easily assume all people are mothers ${ }^{22}$ Moreover, since the universal audience is constructed from experience with real audiences, universal audiences are influenced by the real audiences an arguer encounters.

To sum the discussion so far: the universal audience is a construction of the arguer which focuses on reasonableness, in contrast to other potentially interesting features of real audiences. The question we entered this section with is "what audience could be the one considered in proof development?" The answer did not lie in real or particular audiences. But my goal here is to highlight the positive arguments for saying our audience of interest is the universal audience. The first of these comes from the goals of arguing to the universal audience - it allows the arguer to develop an argument he believes will be permanent as long as reason is permanent. The second similarly deals with the generality of reasoning used in mathematics. It seems related to common-sense reasoning to the mathematician. And this is in line with claims about all reasonable people, not just oneself or mathematicians like oneself.

The final positive argument I will give involves the real audiences mathematicians most often encounter during proof development. We've primarily been focused on the constructed audiences. But arguers benefit from external feedback. They like to know how the argumentation that they give is actually received. For the constructed audience to be useful, it should accept what the actual audience accepts. Arguers construct more faithful particular audiences from experience with their real audiences. But there is no way of receiving external feedback from the universal audience since it is only a construction. So while the argumentation is to the universal audience, it can only be received by real audiences and informed by real and particular audiences. The way to receive external feedback is by arguing to the universal audience's incarnations - the real audiences assumed to be closest representatives of the universal audience. According to [Perelman and Olbrechts-Tyteca(1969)], there are two real audiences which are close incarnations of the universal audience: the audience of oneself and the audience of a single interlocutor. Neither of these incarnations are substitutes for the universal audience because their backgrounds and particularities hinder the abstraction to just reasonableness. But much of the external feedback arguers stand to receive while constructing an argumentation to the universal audience is from these incarnations.

This story of feedback being proffered primarily by the audience of oneself and the single interlocutor is remarkably applicable in mathematics. Math-

\footnotetext{
22 This is a psychological claim which I have neither the room nor the evidence to defend in the specifically mathematical case. This is a limitation I address in the concluding remarks.
} 
ematical proof development with real audiences primarily occurs via selfdeliberation when one tests one's own reactions to a component argument. Next to that is co-authorship where the mathematician tests his component arguments against someone who is as concerned with finding the argument aimed at the universal audience.

Section 2 showed that mathematical proof is typically argumentation and these argumentations necessarily reflect the intended audience. The question remained what is the intended audience in development? Adapting to intended audiences for presentations are clear, i.e. adapting for an introductory course or for journal publication. But teasing out the intended audience in development is more difficult. I proceeded by evaluating 3 types of audiences - real, particular, and universal - against mathematician's descriptions of proof. I argue that the first is not practical for development. The second is not in line with claims of generality or permanence. I argue that the third is in line with mathematician's reflections and the real audiences they receive feedback from.

At this point, the main thesis of the paper is in place. What remains to be shown is what good comes from a theory such as this. If the readers accept the entirety of the paper so far, they may walk away and look at any proof and say "ah the mathematician's universal audience is reflected in that." But these claims are always possible to generate ${ }^{23}$ Since they are always possible to generate about any proof, they do very little by way of illumination. To show that the thesis I've defended here does help us illuminate and explain features of proof, I will turn in 4 to a case study.

\section{A Case Study: 1920s Knot Invariants}

In this section, I will examine a case study to show how the audience-focused framework introduced in Section 3 can be used to illuminate why certain features of proof are used and claimed to be better than others ${ }^{24}$ In 1926, Kurt Reidemeister claimed that he had found the first calculable knot invariants in his papers "Knoten und Gruppen" and "Elementare Begründung der Knotentheorie." In 1927, James W. Alexander and G. B. Briggs claimed they had found the first calculable knot invariants in "On Types of Knotted Curves." They both came to the same conclusions about knot invariants, but their arguments were different. I will argue that parts of Alexander's methods are best

\footnotetext{
23 I'm grateful to an anonymous reviewer for making this point which forced me to get clearer about what the case study provides to this paper. This point entails that looking at proofs themselves will not generate any evidence for the main thesis. But the argument above stands without attempting to draw evidence from these easily generated claims.

24 My case study draws on Epple(2004). Epple's argument looks at how local research becomes more universal mathematical knowledge. I focus on the methods in the proof, in particular Alexander and Briggs' dotting notation, and look at standards of reasonableness abstracted from local particular audiences.
} 
understood in light of his universal audience, which attempts to incorporate the standards of two very different particular audiences he constructed 25

A knot is the embedding of a circle into a three-dimensional Euclidean space. In the 1920s, the basic problem in knot theory was classifying types of knots. A knot invariant is a quantity, often a number, that identifies which knots are equivalent. In Reidemeister's and Alexander's case these were deformation invariants; two knots are equivalent if, in a series of finite steps, they could be deformed into each other. Both Reidemeister and Alexander produced algorithms for calculating knot invariants and both of their algorithms produced the same invariants. In spite of this, the actual algorithms and proofs were mathematically very different. I will thoroughly examine Alexander and Briggs(1927)]'s algorithm and proof. Reidemeister's proofs and algorithm will be used to compare proof methods later in the section, but not as a full case study itself. I will begin by discussing the proofs and algorithms in "On Types of Knotted Curves." In that discussion I will show that they introduce and rely on a dotting notation throughout the paper. In 4.2, I will argue that audience consideration provides an illuminating explanation for its introduction ${ }^{26}$

\subsection{Alexander's Knot Invariants}

Alexander and Brigg's algorithm depends on linear systems and homologies. The algorithm starts with a knot diagram (see Figure 3). Both today and in Reidemeister, crossings are noted with broken lines. The lower branch of a curve has a break while the top branch stays solid at the crossing (see Figure 4). But Alexander and Briggs(1927) choose to use dots when representing crossings (see Figure 3 ). The instructions provided to dot the crossings call for an observer:

We imagine an observer standing on the $x y$-plane and describing the polygon of the diagram once in the positive sense ... thereby passing twice through each crossing point. Then, as the observer passes through a crossing point on the segment representing an upper branch of the knot, we mark with dots the two corners on his right; as he passes through a crossing point on the segment representing a lower branch, we make no notation of any sort. Alexander and Briggs(1927), p. 564]

\footnotetext{
25 I refer to Alexander's universal audience here and afterward. The 1927 paper is coauthored with his student G. B. Briggs. But the 1928 paper is written solely by Alexander. Briggs makes vital contributions, but I take these facts - that Briggs was his student and Alexander published a later paper on it alone — as evidence that Alexander is mostly the one determining standards. Epple(2004)] also relies on Alexander's background but not Briggs's.

26 For more theoretical work on the importance of knot diagrams as evidence, see De Toffoli and Giardino(2014)].
} 


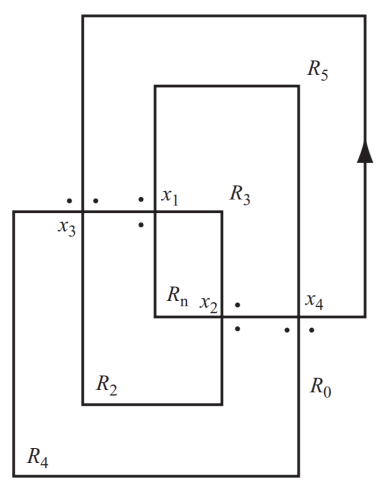

Fig. 3 One of Alexander and Briggs(1927)]'s knot diagrams.

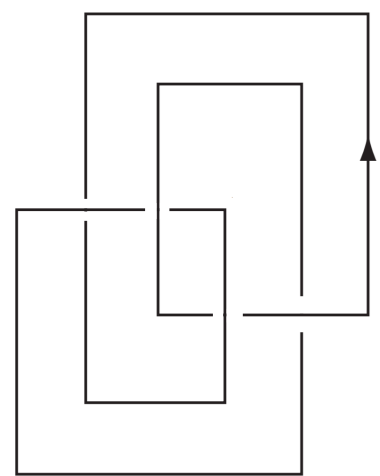

Fig. 4 Alexander and Briggs(1927)'s representation of the broken line notation.

This dotting notation turns out to be vital to their later proofs regarding knot invariants. In [Alexander(1928)], the observer is replaced by an ant but it remains in Alexander's work on knot invariants 27

After constructing the plane diagram, Alexander and Briggs transform the knot into linear systems and homologies. This produces the algorithm for calculating the invariants. The linear system $X$ is the set of all finite linear combinations of uninterpreted marks. For a set of $k$ arbitrary marks from the system $X$, the set of all linear combinations of $y_{s}$ where

$$
y_{s}=\sum_{i=1}^{m} \epsilon_{s i} x_{i}, \quad s=1,2, \ldots, k
$$

defines a linear subsystem $Y$ of $X$. Any two elements $x, x^{\prime}$ in $X$ are homologous, $x \sim x^{\prime}(\bmod Y)$ iff the difference $x-x^{\prime}$ was in $Y$. The $y_{s} \sim 0$ are called fundamental homologies. A plane diagram with $\nu$ crossings is given $n \nu$ marks

27 The dotting notation is only mentioned once by Epple as a "seemingly marginal difference" between Reidemeister and Alexander and Briggs Epple(2004). 
for some integer $n$. Then, for every region of the diagram, $n$ fundamental homologies are defined using the marks associated with the corners of that region and coefficients $\epsilon_{s i}$ depending on the over and undercrossings denoted by dots in the diagram. A matrix is generated with the $\epsilon_{s i}$. This matrix's elementary divisors greater than one were the torsion numbers of the knot. Alexander and Briggs give one example for $n=2$ and the knot diagram in Figure 3 .

Following the algorithm are a number of proofs regarding the torsion numbers of knots and their inverses and reflections. These rely heavily on the dotting notation chosen earlier. For example, the inverse of a knot diagram is defined by the shifting of dots — not abstract mathematical definitions. In addition, proofs rely on recognizing certain facts about how the shifting of dots changes the knot. Take, for example, the theorem that the homologies determined by any two regions with consecutive indices $k$ and $k+1$ are consequences of the homologies determined by the remaining $\nu$ regions of the diagram. The first step of the proof is observing that "at each crossing point, $x_{\alpha}$, there are two dotted and two undotted corners and that one corner of each sort belongs to a region of odd index, the other to a region of even index" Alexander and Briggs(1927), p. 574]. This observation leads to the conclusion that sets of identities, $H_{s}$, where $\sigma$ is the number of regions in the plane diagram

$$
H_{s}=\sum_{\sigma} \epsilon_{\sigma} y_{\sigma s} \sim 0
$$

will actually be sums of groups of terms of the form

$$
x_{\alpha s}-x_{\alpha s}+x_{\alpha(s+1)}-x_{\alpha(s+1)} \sim 0
$$

and will vanish identically. No more evidence for this conclusion is provided than the observation about the dots. But they think no more is needed to convince their intended audience. Seeing as the goals of proof are in line with the goals of argumentations directed at the universal audience, then Alexander and Briggs might think the universal audience will accept this component argument. Mizar and other formal systems would require more justificatory steps than that to show that the identities vanish. This fact about $H_{s}$ is later used in the whole proof of the theorem. But we can see informal reasoning in this step alone. This is an example of the standards of reasonableness that Alexander and Briggs had in their universal audience.

After these smaller theorems about torsion invariants, they go on to give the most important proof in their article: that the torsion numbers of a knot are topological invariants. To establish the truth of this, they show that for each of the four classes of elementary deformation, the torsion number does not vary. They continue to give possible combinations of dots as reasoning in the proof. For example, the result of one of the deformations is that "the corner of the triangle $x_{0} x_{1} x_{2}$ at the crossing point $x_{0}$ is not dotted but that the opposite corner at $x_{0}$ is" Alexander and Briggs(1927), p. 577]. This is then used to show that the system of marks and homologies after deformation reduces to the system of marks and homologies before deformation. 
At the close of the paper, Alexander and Briggs give a discussion of how the torsion numbers of a knot may be interpreted in terms of "Betti numbers and coefficients of torsion of an $n$-sheeted covering $J_{n}$ with a branch curve of order $n-1$ covering the knot" Alexander and Briggs(1927), p. 577]. Epple(2004) interprets this section of the paper as the true start of the mathematical reasoning. It shows how the mathematicians abstracted from known work using cell decompositions inspired by Poincaré's work. They began this work with this approach but in the end they used uninterpreted marks which we saw throughout the paper. This is important in 4.2 , since it suggests that, at root, the research was inspired by Poincaré.

The discussion so far indicates two things (1) the dotting notation is an innovation and (2) the dotting notation is relied upon in proof. In 4.2 , the aim will be to show that Alexander's comments, combined with the dotting notation, are best explained by the thesis that Alexander developed his proof to his universal audience.

\subsection{Introducing the Dotting Notation}

We saw that the dotting notation was a vital part of Alexander's work and breaks with the typical notation seen in knot diagrams. Alexander was not ignorant of the typical notation, as he addresses it in his paper. In fact, he claims that his dot notation is better for his purposes Alexander and Briggs(1927), p. 564]. They do not explain why this is the case ${ }^{28}$ Moreover, the current state of knot diagrams does not favor Alexander's better notation, even when parts of his methods are used. Its invention, introduction, and superiority claim can be fruitfully explained by appealing to Alexander's universal audience. In the rest of the section I will argue that Alexander's experiences with particular audiences lead to a universal audience which would best be satisfied by appeals to a dotting notation.

Alexander's universal audience is heavily influenced by two main groups ${ }^{29}$ Alexander has two main particular audiences he has constructed: the axiomatic Hilbertian one and the intuitive Poincarean one. The first audience corresponds to a real audience he has encountered in his daily work; they are his colleagues and superiors. Although Alexander's dissertation work was supervised by Thomas Grunwall, Alexander's earliest work was guided by Oswald Veblen. Veblen was "the main American propagator of Hilbertian ideas" Epple(2004), p. 156]. So the start of Alexander's formation of his universal audience was

28 One excellent objection, worth briefly sketching, to this involves the concept of knots. The objection provides an explanation for the use of "better" by Alexander. It is that the dotting notation introduces direction into the concept of knots. And that Alexander and Briggs were claiming superiority because adding directedness made for a better understanding of knots. Plausible as that may be, even the diagrams with the broken line notation indicate directedness in Alexander and Briggs' paper. So it's not likely that they were adding directedness.

29 The biographical and historical picture gestured at in this section follows Epple(2004). account. 
with Veblen. He would have taken a high level of abstraction as a standard of proof. Alexander would have learned that if a proof was to convince anyone, it would need to be symbolic and abstract. But the work he primarily dealt with is heavily reliant on Poincaré's work. He learned different standards there. Alexander would have constantly found himself imagining the real audiences who would have accepted Poincaré's proofs. In this way, he constructs a particular audience for Poincaré's work. He must have been able to do so in order to be successful at shoring up Poincaré's "rather vaguely justified claim that two different cell decompositions of the same manifold gave rise to the same invariants of (reduced) homology" Epple(2004), p. 157]. From his earlier work, Alexander learned what standards of reasonableness underlied both the Poincarean and Hilbertian audiences.

With this example, more concrete exposition on reasonableness and the universal audience can be provided. Alexander, to construct a universal audience, looks for standards of reasonableness that are active in all his particular audiences. In looking at his two different particular audiences, Alexander recognizes that the reasons accepted by one audience are not accepted by the other, or at least with hesitation. Neither audience is unreasonable, so the conceptions of reasonableness embodied in their particular audiences are different. This is where constructing the universal audience works to extract what methods for evaluating reasons are common between them. His argumentations, in order to be successful, had to primarily be based on these standards. Alexander has constructed his universal audience from the abstracted standards that underlie both of them. This, I argue, carries over into his work on knot invariants and results in the dotting notation.

Through the use of these dots, Alexander is bringing the standards of the Poincarean audience and the Hilbertian audience together. By relying on the dot notation in his proof, he has developed a reason that he believes all people must accept. This reason reflects some more common-sense reasoning between them. It's important to note that Alexander is not just striking a middle ground between two particular audiences. If he were, then recalling the discussion about common-sense truisms, he would view the two audiences as having two different sets of truisms ${ }^{30}$ This seems problematic. But Alexander, in finding the underlying common-sense rule governing both of their reasoning, can use that rule as one defining his universal audience. In this way, the non common-sense standards of the Hilbertian remain with that particular audience; while the truisms common between them are abstracted into the universal audience. Without employing the universal audience, the analysis of this case results in Alexander's proof methods as one that can be read in two different ways - just methods for convincing two particular audiences with seemingly irreconcilable reasoning practices. The dotting notation inferences in this view are an ambiguous tool which can be read two different ways, instead of as a common reason. It's more in line with previously stated features

\footnotetext{
30 Recall that the view expressed by Hadamard is most likely too extreme. But the less extreme claim involving generality of reasoning that it expresses is really what's at play in arguments for the universal audience.
} 
of proof - generality and universality - that a 'better proof method' be one that satisfies those features. Finding a proof method which satisfies two audiences by identifying a deeper reason, then, is better than a proof method which satisfies two audiences by identifying a particular way that both, and potentially no others, can read according to their preferences.

Alexander's choices are mentioned in his 1928 paper where he writes:

To make our descriptions a trifle more vivid we shall often allow ourselves considerable freedom of expression, with the tacit understanding that, at bottom, we are really looking at the problem from the combinatorial point of view. Thus, we shall sometimes talk of a knot as though it were a smooth elastic thread subject to actual physical deformations. There will, however, never be any real difficulty about translating any statement that we make into the less expressive language of pure, combinatorial analysis situs. In the figures, we shall picture a knot by a smooth curve rather than by a polygon. A purist may think of the curve as a polygon consisting of so many tiny sides that it gives an impression of smoothness to the eye [Alexander(1928), p. 276].

This discussion is not about the dotting notation in particular. It is about his decision to use smooth curves instead of polygons in his knot diagrams. But the quote reflects on an overwhelming theme - one that encompasses both the dotting notations and the smooth curves - in Alexander's work on knot theory. In his 1927 paper, Alexander doesn't give any such discussion. He claims these freedoms without justification. If Alexander was primarily arguing to his universal audience, he would not have felt such justification was needed. If Alexander thought he was writing to two distinct audiences, with a method that could be read both ways, he should have provided this justification from the start. His 1928 paper shows changes on that front. The need he feels to justify his choices is new and reflects a recognition that not all persons would accept his methods. It reflects the realization that his original audience was not genuinely universal. But he didn't realize this at first. In the 1927 paper, no similar gloss is needed. This can be explained by Alexander's belief that his method was more acceptable, and not just a method that could be read two different ways. But he does not change his methodology in the 1928 paper, he keeps the methodology but adds a gloss for the particular, objecting audience. I take this as further evidence that the methods were not intended as inferences which can be read both ways. They were intended as a method identifying a common inference between the two particular audiences, and many others. Another important point here is that the dotting notation is not completely vital to the methodology. The fact that Alexander thinks the problem has combinatorial underpinnings means that filling out the details, instead of using the dots, seems plausible. The dotting notation is not just a requirement of the method. It's a piece of the method, where the decision to include it was a considered one.

The final point of analysis for this case study returns to Reidemeister's work. Alexander and Briggs's paper was published with a priority claim on 
what was essentially the same work by Reidemeister. Epple(2004)] shows that Reidemeister and Alexander and Briggs worked in local traditions. By working in these traditions, Alexander and Briggs at Princeton, and Reidemeister in Vienna, were trained in different approaches. But it is not just that the two employ different well-established methods and notations. Alexander introduces a notation and heavily relies on it in his argumentation. The argument I've given in 2 and 3 allows us to explain this by appeal to Alexander's universal audience which required an identification of the standards that underlied Poincarean audiences and Hilbertian audiences. Reidemeister does not introduce and use a dotting notation. Again, we can reconstruct this choice by looking at Reidemeister's universal audience. It was heavily influenced by the Vienna Circle to the point that this influence outweighed any last particular influence from Poincaréan standards. In particular, while Alexander was in a local tradition of professionalization and that influenced his formal standards, Reidemeister's formal standards were not just mathematically driven but philosophically driven. Reidemeister's universal audience was satisfied without a dotting notation. So he did not need to introduce one.

My arguments are limited by the fact that we are examining finished products. The published work of Alexander only allows us glimpses into his mind during the development of the proof. I have argued that parts of his work can be usefully reconstructed as influenced by his attempt to argue to his universal audience. In particular, the introduction of the dotting notation represents a recognition and use of standards in two particular audiences. Because component arguments to the universal audience are supposed to convince every reasonable person, this explains Alexander's seemingly unsubstantiated claim that the dotting mechanism is better. Alexander and Briggs's work is a concrete example of mathematical practice that audience analysis sheds a light on. Again, this case study does not provide evidence for the thesis that mathematicians are arguing to their universal audiences when developing proof. Rather, it provides evidence that once accepting such a thesis, on the basis of preceeding arguments, it is explanatorily useful.

\section{Concluding Remarks}

The role of audiences in mathematical proof has largely been neglected, in part due to misconceptions originating in Perelman and Olbrechts-Tyteca(1969) which bar mathematical proofs from bearing reflections of audience consideration. Typically, mathematical proof is argumentation, not derivation. I've argued that a mathematician develops a proof with his universal audience in mind. In so doing, he creates a proof which reflects the standards of reasonableness embodied in his universal audience. Given this framework, we can fruitfully reconstruct the introduction of proof methods based on the mathematician's likely universal audience, as seen in Alexander's dotting notation.

There are a number of limitations to the arguments I've provided here. One such limitation is that it heavily relies on a psychological claim about what 
it's like to use the constructed audience in mathematics. It's assumed that this parallels the use in argumentation. But this claim should be tested empirically. This is an important future project for bolstering the claims made in this paper. Moreover, I've argued that proof development is audience reflective. But this is not all there is to proof. There are other normative and objective standards to be considered for proof. I think further examination of mathematical audiences will help to shed light on how the standards interact. As such, I want to conclude this paper with some open problems and potential directions. A thorough account of how the universal audience is constructed, from education to advanced research, is needed. The adaptation of established work to particular audiences also remains to be explored. Such an account would help us understand mathematical explanation to non-mathematicians. It also remains to be seen how historical changes in mathematical practice are related to universal audiences. As in Alexander's case, the introduction and uptake of new techniques, methods, and concepts are better understood by adding audience consideration. Finally, the framework explored here introduces the possibility that audience influence plays some role in what constitutes acceptable proof methods. This means that a thorough understanding of what mathematical proof and rigor are, calls for further inquiry into the intended audiences of proof.

Acknowledgements I am incredibly grateful for the comments from (and conversations with) Andrew Aberdein, Nic Fillion, Tom Archibald, Silvia de Toffoli, two anonymous referees, and members of the audience at the CSHPS 2017, CSHPM 2018, the 2019 Masterclass on Philosophy of Mathematical Practice, and the 2020 APMP meetings. Although they will not agree with all of the arguments and conclusions in the paper, their comments greatly improved the manuscript.

\section{References}

Aberdein(2019). Aberdein A (2019) Evidence, Proofs, and Derivations. ZDM Mathematics Education 51(4)

Aberdein and Dove(2013). Aberdein A, Dove IJ (eds) (2013) The Argument of Mathematics. Springer, Dordrecht

Alexander(1928). Alexander JW (1928) Topological Invariants of Knots and Links. Transactions of the American Mathematical Society 30(2):275-306

Alexander and Briggs(1927). Alexander JW, Briggs GB (1927) On Types of Knotted Curves. Annals of Mathematics 28(1):562-586

Antonutti Marfori(2010). Antonutti Marfori M (2010) Informal proofs and mathematical rigour. Studia Logica 96:261-272

Azzouni(2004). Azzouni J (2004) The derivation-indicator view of mathematical practice. Philosophia Mathematica 12(2):81-105

Azzouni(2009). Azzouni J (2009) Why do informal proofs conform to formal norms? Foundations of Science 14(1-2):9-26

Carrascal(2015). Carrascal B (2015) Proofs, mathematical practice and argumentation. Argumentation 29(3):305-324

Corcoran(1989). Corcoran J (1989) Argumentations and Logic. Argumentation 3:17-43

Davis and Hersh(1986). Davis PJ, Hersh R (1986) Mathematics and rhetoric. In: Davis PJ, Hersh R (eds) Descartes' Dream: The World According to Mathematics, Penguin, London, pp 57-73 
De Toffoli and Giardino(2014). De Toffoli S, Giardino V (2014) Forms and roles of diagrams in knot theory. Erkenntnis 79(4):829-842

Dove(2009). Dove IJ (2009) Towards a theory of mathematical argument. Foundations of Science 14(1-2):137-152

Dufour(2013). Dufour M (2013) Arguing Around Mathematical Proofs. In: Aberdein A, Dove IJ (eds) The Argument of Mathematics, Springer, Dordrecht, pp 61-76

Epple(2004). Epple M (2004) Knot Invariants in Vienna and Princeton during the 1920s: Epistemic Configurations of Mathematical Research. Science in Context 17(1):131-164

Fallis(2003). Fallis D (2003) Intentional Gaps in Mathematical Proofs. Synthese 134:45-69

Hadamard(1945). Hadamard J (1945) An Essay on the Psychology of Invention in the Mathematical Field. Princeton University Press, Princeton, NJ

Hadamard(2004). Hadamard J (2004) Thoughts on the heuristic method. In: Ayoub RG (ed) Musings of the Masters, The athematical Association of America

Hardy(1929). Hardy GH (1929) Mathematical proof. Mind 38:1-25

Hume(1739). Hume D (1739) A Treatise of Human Nature. Penguin Books, London, 1969 Reprint

Krantz(2011). Krantz SG (2011) The Proof is in the Pudding: The Changing Nature of Mathematical Proof. Springer, New York, NY

Larvor(2016a). Larvor B (ed) (2016a) Mathematical Cultures: The London Meetings 20122014. Birkhäuser, Basel

Larvor(2016b). Larvor B (2016b) Why the naïve derivation recipe model cannot explain how mathematicians' proofs secure mathematical knowledge. Philosophia Mathematica 24(3):401-404

Line Andersen(Forthcoming). Line Andersen HS Mikkel Johansen (Forthcoming) Mathematicians writing for mathematicians. Synthese

Perelman and Olbrechts-Tyteca(1969). Perelman C, Olbrechts-Tyteca L (1969) The New Rhetoric: A Treatise on Argumentation. University of Notre Dame Press, Notre Dame, IN

Sigler(2015). Sigler J (2015) The new rhetoric's concept of universal audience, misconceived. Argumentation 29(3):325 - 349

Tanswell(2015). Tanswell F (2015) A problem with the dependence of informal proofs on formal proofs. Philosophia Mathematica 23:295-310

Tindale(2015). Tindale CW (2015) The Philosophy Of Argument And Audience Reception. Cambridge University Press, Cambridge

Wiedijk(2004). Wiedijk F (2004) Formal Proof Sketches. In: Berardi S, Coppo M, Damiani F (eds) Types for Proofs and Programs: International Workshop, TYPES 2003, Torino, Italy, April 30 - May 4, 2003, Revised Selected Papers, Springer, Berlin, LNCS, vol 3085 , pp 378-393 